\title{
Road Boundary Detection in Complex Urban Environment based on Low- Resolution Vision
}

\author{
Qinghua Wen, Zehong Yang, Yixu Song, Peifa Jia \\ State Key Laboratory on Intelligent Technology and Systems, Tsinghua National Labora- \\ tory for Information Science and Technology, Department of Computer Science and \\ Technology, Tsinghua University, Beijing, 100084, China \\ Email:wenqh06@mails.tsinghua.edu.cn, yangzehong@sina.com, \\ songyixu@sohu.com,dcsjpf@mail.tsinghua.edu.cn
}

\begin{abstract}
In this paper, we proposed a real-time road boundary detection method in complex urban road environment. The detection difficulty lies in road wear, both existence of marked and unmarked boundary and low-resolution vision. The idea of the algorithm is to extract the road surface firstly using improved region growing method based on edge enhancement. The road boundary is then estimated by fitting the edges of the extracted road surface. A Bezier splines algorithm with optimization of control points is proposed to estimate the road boundary. The algorithm is implemented on the video collected in BEIJING urban streets and achieves good performance.
\end{abstract}

Keywords: driving assistance system, road boundary detection, lane detection, region-growing, Bezier spline

\section{Introduction}

It is assumed that up to $90 \%$ car accidents are caused by driver faults [1]. So driving assistance system which could work as extended eyes to help the driver to perceive the blind area in the road and as early warning to remind the driver the potential danger had become a hot topic since 1990s. In the driving assistance systems, road detection is a key and indispensable issue because road boundary defines the drivable safe-driving area in both unmarked and marked road and often performs as the preliminary stage for further obstacle detection and tracking. Road boundary and lane marking detection are collectively called road boundary detection for short in the rest of this paper.

Many sensors such as radar and laser had been used to perceive the road scene. But vision-based camera is thought can capture more information than other sensors. So vision-based, either monocular vision or stereo vision, road boundary detection has been received a lot of attention. The road boundary detection in structured road had been well-researched [2][3]. The current work mainly focus on more complex urban environment in which the influence factors include wear, crossroads, the occlusion of vehicle, shadows, illumination effects, signs on roads and so on. The complex road condition makes it difficult to design a general detection algorithm which can deal with all of these problems. So some literatures deal with specific road scene instead [4].

Road boundary detection generally can be divided into two steps. The first step is segmentation which gets the position of lane in the image. Thresholding color of 
the image after filtering and image transformation such as IPM is common used but only fits in clear road [1]. Clustering and classification method is often used in unmarked road [7][8][10]. Some constraints are often assumed to limit the road condition. Utilizing the priori knowledge is supposed useful in this step. The second step is representation which is to state the true position of the road boundary in the image. The simplest way is straight line model using Hough Transform. Other models include hyperbola model [5], parabola model [6] and clothoid model. This step may correct the error brought from the first step.

In this paper, we proposed a method for typical urban road streets. For the reason of energy and price cost, the lowresolution camera is adopted to record the road scene. The difficulty of detection in the road scenario includes (1) road wear. (2) existence of both marked and unmarked boundary. (3) low-resolution vision. An example of the road video as follows.

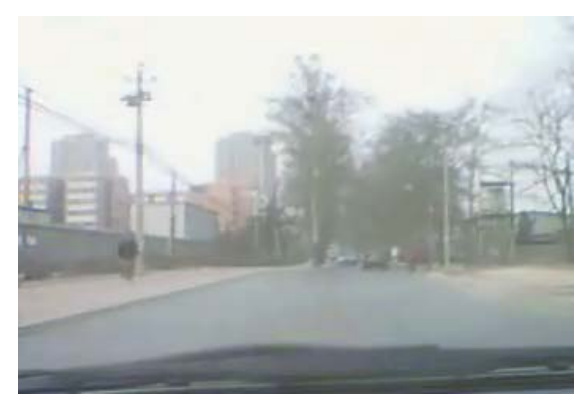

Fig. 1: Example of the road video

The rest of the paper is organized as follows. In Part 2, we first analyze the problem and introduce the basic principle of the proposed method, then present the method in detail. In Part 3, the experiment results of the algorithm running on different road environments are presented as well as some analysis of the effectives of the proposed method. In the end, the conclusion and future work are presented.

\section{Proposed method}

By analyzing the target video, we can find edge-based method is unfeasible Because of the wearing boundary. The not necessary existence of boundary marking also make the algorithm relied on the color high-contrast between the marking and its surrounding invalid. The existence of both marked and unmarked boundary makes the algorithm proposed should be fit to both conditions without assuming the either one in advance. In another aspect, the low-resolution vision makes the algorithms relied heavily on thresholding image color invalid. But the advantage brought from low-resolution image is to make the color of road surface seem consistent, as shown in Fig. 1.

The proposed algorithm is based on the color consistency of the road surface. We first compute the road surface color and its variance by assuming the road color obeys to Gaussian distribution, and then extract the road surface using an improved region growing method with edge enhancement. In the end the edges of the road surface are fitted as the road boundary using Bezier spline algorithm with optimization of control points.

\subsection{Preprocessing}

For the task of road boundary detection, we don't need deal with the whole region of image. Using the height of the camera from the ground and its yaw angle and pitch angle, we can estimate the vanishing point of the road approximately. To reduce computation consumption and to remove unnecessary noise, then we determinate the region of interest (ROI) from the bottom of the image to the vanishing point of the road for further road boundary detection. For the examined 
$352 * 240$ video, our ROI is the $352 * 50$ area as in Fig. 5.

\subsection{Estimating road surface color}

In the asphalt road, the road surface is approximately gray, and the difference of its red, green, blue component are considered less than 25 [8]. The lowresolution vision also makes the hue contrast of different object in the image not clear. So it is enough to only cope with its gray image for the sake of reducing computing time cost. The gray image is first smoothed by Gaussian filter. Assuming the intensity of the pixels of the road surface is subject to Gaussian distribution. We use $\hat{\mu}_{n}$ and $\hat{\sigma}_{n}$ represent the mean and variance of the Gaussian distribution in frame $n$

$$
P(x)=\frac{1}{\sqrt{2 \pi} \sigma_{n}} e^{-\frac{\left(x-\mu_{n}\right)^{2}}{2 \sigma_{n}^{2}}}
$$

The $\hat{\mu}_{n}$ and $\hat{\sigma}_{n}$ are taken as the road surface color and its variance.

Since the road surface is just part of the image. We don't need to compute the whole image. The change between adjacent frames is slight, so we take the estimated road surface area of the frame $n-1$ as the part for compute the Gaussian distribution in frame $n$. For the first frame, we take the triangle area in the middle of the image which is the conservative estimate of the road surface for the most frames as the initial road surface as illustrated in Fig. 2.

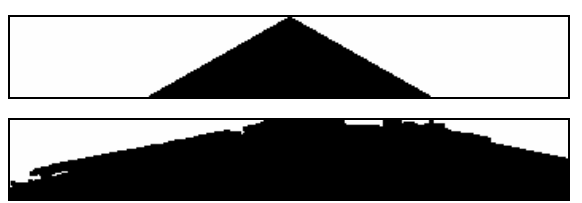

Fig. 2 Up: the mask of computing area for the first frame. Bottom: the road surface area of the frame $n-1$ as the mask of computing area for frame $n$
The $\mu_{n}$ and $\sigma_{n}$ can be computed by the maximum likelihood estimate procedure. Which yield

$$
\begin{aligned}
& \hat{\mu}_{n}=\frac{1}{m-1} \sum_{(i, j) \in \Omega} I(i, j) \\
& \hat{\sigma}_{n}=\frac{1}{m-2} \sum_{(i, j) \in \Omega}\left(I(i, j)-\hat{\mu}_{n}\right)^{2}
\end{aligned}
$$

Where $\Omega$ is the mask area defined by Figure 3 , and $m$ is the total pixel number of the area $\Omega$.I is the intensity of the pixels.

\subsection{Extracting road surface area}

\subsubsection{Enhancing road edge}

Enhancing image edge, including road edge, is a critical step in the proposed method. Two kind of edge are extracted: the line edge and non-line edge. For the line edge, the Probabilistic Hough Transform is used. For the non-line edge, the firstorder Sobel operator is employed. We only enhance the edge of the non-road surface area. The two edges are added to the original image.

The aim of enhancing road edge is to make the wearing boundary more present and visible, so as to extract the road surface correctly in the Road surface extracting module. An example comparing the detection result with and without enhancing road edge step as follows.

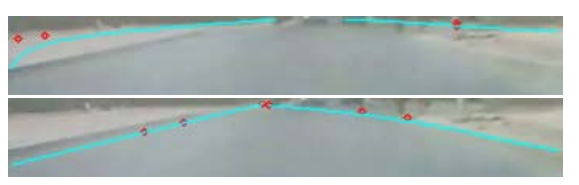

Fig. 3 Top: without the enhancing road edge. Bottom: with the enhancing road edge step

\subsubsection{Road surface extraction}

After enhancing the edge, the region growing method is employed to extracting the road surface. Region growing method groups the pixels which have the 
same property to form a connective region, starting from a seed point.

There are two initial seed points, $a$ and $b$, which adaptively selected in the middle top and bottom of the image respectively. $a$ and $b$ satisfy $\hat{\mu}_{n}-\hat{\sigma}_{n}<a, b<\hat{\mu}_{n}+\hat{\sigma}_{n}$, otherwise, the nearest neighbor pixels around $a$ and $b$ which satisfy the condition to replace them. The two connective regions starting from these two seeds mix together as the road surface.

There are two criteria work as the end condition of region growing. They are

$$
\left\{\begin{array}{l}
\left|I_{\text {seed }}-I_{\text {neighbor }}\right|<\gamma \\
\left|I_{\text {neighbor }}-\hat{\mu}_{n}\right|<\hat{\sigma}_{n}
\end{array}\right.
$$

Where $I_{\text {neighbor }}$ is the intensity of the adjacent pixel of the seed point, $\gamma$ is the difference limit of the adjacent pixel pair. $\hat{\mu}_{n}, \hat{\sigma}_{n}$ are the road surface color and its variance determined in the 2.1. step.

\subsection{Estimating road boundary}

The previous steps have give us the candidate road surface, which firstly denoised by morphological operations in this step. The third degree Bezier spline using Bernstein polynomials [9] is then used to fit the left and right edge of the road surface, which is supposed as the true road boundaries. The advantage using Bezier spline lies on it can fit arbitrary shape of the curve with enough control points. It is incomplete to assume the predefined model [5] of the road boundary in the unstructured road such as urban or campus streets.

The third degree Bezier spline is defined by four control points and is a cubic polynomial as

$$
\begin{aligned}
P(t) & =(1-t)^{3} P_{0}+3 t(1-t)^{2} P_{1}+3 t^{2}(1-t) P_{2}+6 t^{3} P_{3} \\
& =\left(t^{3}, t^{2}, t^{1}, 1\right)\left(\begin{array}{cccc}
-1 & 3 & -3 & 1 \\
3 & -6 & 3 & 0 \\
-3 & 3 & 0 & 0 \\
1 & 0 & 0 & 0
\end{array}\right)\left(\begin{array}{c}
P_{0} \\
P_{1} \\
P_{2} \\
P_{3}
\end{array}\right)
\end{aligned}
$$

Where is to control the fitting fineness. $P_{0}, P_{1}, P_{2}, P_{3}$ are the control points. $P(0)=P_{0}, P(1)=P_{3}$, the interior points $P_{1}, P_{2}$ control the shape of the spline.

We fit the Bezier splines using leastsquared error method. For the left boundary, given the point series sampled from the left edge of the road surface $\left\{P_{0}, P_{1}, \cdots, P_{n}\right\}$, the number of points determined by the sampling precision. Let the control point $P_{0}$ set as the point $p_{0}$, the control point $P_{n}$ set as the point $p_{n}$. Only the two interior points $P_{1}, P_{2}$ is variable points, they are selected in the rest edge points $\left\{P_{1}, \cdots, P_{n-1}\right\}$ by minimizing the error between the original boundaries with the Bezier spline fitted by $P_{0}, P_{1}, P_{2}, P_{3}$. Here the original boundary denotes the left edge of the road surface. The error is measured by the area formed by the original boundary and the Bezier spline as shown in Fig.4. To avoid time-consuming integral calculus, it approximately estimated by counting the pixels between the original and fitted boundary. The point series $\left\{p_{0}, p_{i}, p_{j}, p_{n}\right\}, 0<i, j<n$ with the least pixel count by minimize (5) are taken as the optimal control points.

$$
A(i, j)=\sum_{\Omega_{i} \in \Omega} \operatorname{Count}\left(\Omega_{i}\right)
$$

Where $\Omega$ is the whole area between the original boundary and the fitted spline $R$.

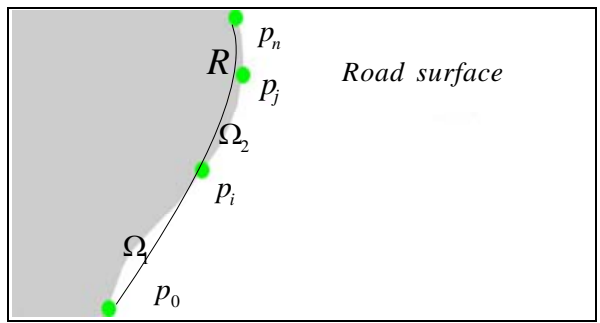

Fig. 4: Bezier spline fitting algorithm 


\section{Experiment and analysis}

The algorithm is run on three video clips which are collected from BEIJING

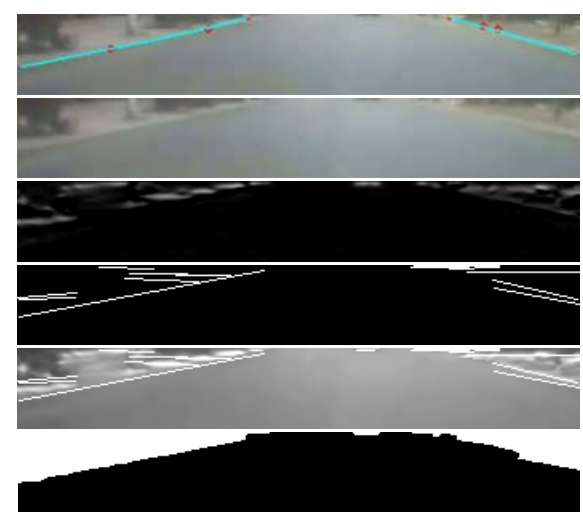

Fig. 5: Result of road boundary detection in a straight road. From up to bottom: ROI with detection result, gray image after smoothing, non-line image edge, line image edge, gray image after enhancing image edge, the mask of road surface

urban streets. The Clip \#1 scenario is the straight road with wearing and unmarked boundary, the Clip \#2 is the unmarked curve road, and the Clip \#3 is the marked scenario with crowd vehicle. Fig. 6 shows the robustness of the algorithm in the supposed road scenario.

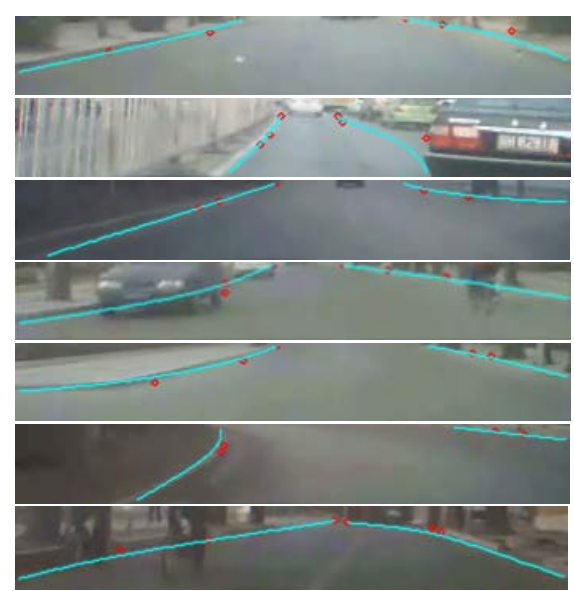

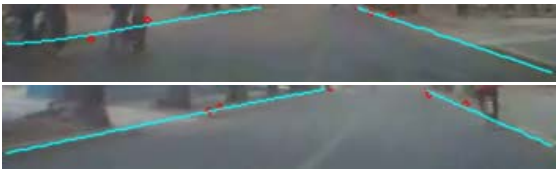

Fig. 6: Detection samples show robustness in wearing boundary, unmarked road, marked road, in presence of vehicle and curves

Fig. 7 shows some false detection. In two conditions, the false detection occurs. They are (1) a majority of the road boundary is occluded by vehicles or pedestrian, (2) a majority of the road surface is occupied by illumination area. For the first case, however, it is reasonable to take the outside of the vehicle as the boundary because the true boundary is invisible and the area occupied by the vehicle is not drivable area. For the second case, an improved region growing algorithm which is invariant to shading and highlight effects using the Mixture of Principal Components algorithm [11] can be introduced to tackle this problem, this is part of our future work.

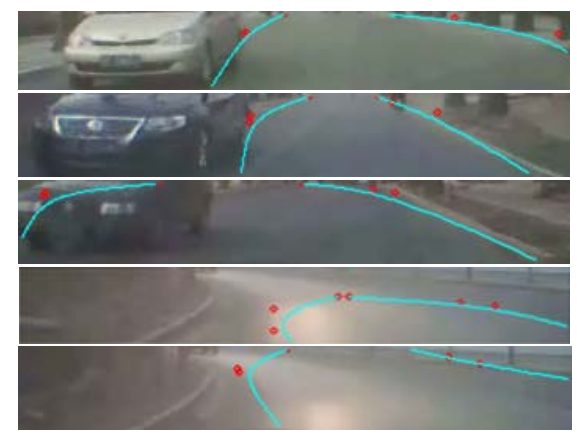

Fig. 7: Examples of false detection

Table 1: Average computational time

\begin{tabular}{llll}
\hline Test Module & Clip\#1 & Clip\#2 & Clip\#3 \\
\hline $\begin{array}{l}\text { Average time per } \\
\text { frame (ms) }\end{array}$ & 18.70 & 15.39 & 31.44 \\
\hline
\end{tabular}

This algorithm was implemented in $\mathrm{C}++$ using the open source OpenCV library on PC with Intel P4 CPU $3.0 \mathrm{GHz}$ 
and 2GB memory. For the examined $352 * 50$ video, the average computational time per frame is less than $25 \mathrm{~ms}$. Table 1 lists the average computational times for the three test video clips. The time cost is proportional to the road surface area.

\section{Conclusion and future work}

In this paper, a road boundary detection solution is proposed for the complex urban environment characterized by wearing edge and both marked and unmarked boundary on low-resolution video with monocular. The experiment on the real road video shows the robustness of the method.

The future work include: (1) Advanced region growing algorithm considering the illuminate effect will be introduce to remove illuminate effect. (2) Since this method only detects the road boundary, but the dashed lane marking, if any, in the middle of the road surface is useful to road understanding, the lane marking detection under known road boundary is also a future work. (3) The non-road area between the detected left and road boundary can be classified as obstacles (road marker, vehicles, pedestrians etc). The further classification and tracking of this non-road area will be a useful work for road understanding.

\section{Acknowledge}

This work is supported by The National High Technology Research and Development Program of China.

\section{References}

[1] Mohamed Aly, "Real time Detection of Lane Markers in Urban Streets," http://www.vision.caltech.edu/malaa /publications /aly08realtime.pdf

[2] K. Kluge, "Extracting Road Curvature and Orientation From Image
Edge Points Without Perceptual Grouping Into Features," Proceedings of the Intelligent Vehicles Symposium, pp 109-114, 1994.

[3] C. Kreucher, S. Lakshmanan, “A frequency domain approach to lane detection in roadway images," Proceedings of the International Conference on Image Processing 1999, Volume 2, pp. 31-35, 1999.

[4] Hsu-Yung Cheng, Bor-Shenn Jeng. "Lane Detection With Moving Vehicles in the Traffic Scenes," IEEE Transactions on intelligent transportation systems, VOL. 7, NO. 4, pp.571-582, 2006

[5] W. N. Zhu, "Lane Detection in Some Complex Conditions," Proceedings of the 2006 IEEE/RSJ International Conference on Intelligent Robots and Systems, pp.117 122, 2005.

[6] K. Y. Chiu and S. F. Lin, "Lane Detection using Color-Based Segmentation,” Intelligent Vehicles Symposium, 2005, pp.706 - 711, 2005

[7] J. D. Crisman and C. E. Thorpe, "SCARF: A color vision system that tracks roads and intersections," IEEE Trans. Robot. Automation, vol. 9, pp.49-58, 1993.

[8] J. Crisman and C. E. Thorpe, "UNSCARF: A color vision system for the detection of unstructure roads," Proc. IEEE Int. Conf. Robotics and Automation, pp. 2496-2501,1991

[9] David Solomon, "Curves and Surfaces for Computer Graphics,” Springer, 2006.

[10] Ola Ramstrom and Henrik Christensen, "A method for following of unmarked Roads," Intelligent Vehicles '05, pp.650 - 655, 2005.

[11] Slawo Wesolkowski and Paul Fieguth, "Color Image Segmentation Using a Region Growing Method,” http://www.iscc.org/aic2001/abstrac ts/oral/Wesolkowski_Fieguth.doc 
Proceedings of the 11th Joint Conference on Information Sciences (2008) Published by Atlantis Press

$$
\text { (C) the authors }
$$

